\title{
Climate conditions and work-related fatigue among professional drivers
}

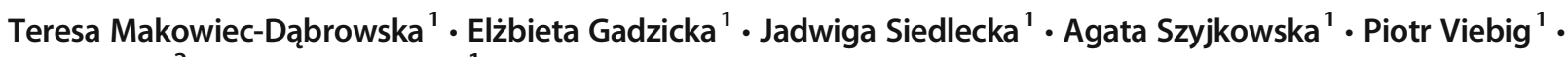 \\ Piotr Kozak ${ }^{2} \cdot$ Alicja Bortkiewicz ${ }^{1}$
}

Received: 8 February 2018 / Revised: 28 September 2018 / Accepted: 29 October 2018 / Published online: 8 November 2018

(C) The Author(s) 2018

\begin{abstract}
The possible associations between climate parameters and drivers' fatigue have not been subject to specific studies thus far. We have undertaken a study to investigate whether the particular climate parameters are related to fatigue perception by motor vehicle drivers. The study was performed from July to October. Each driver was surveyed four times: before and after workshift on a monotonous route outside the city center (MR), and on a heavy traffic route in the city center (HTR). The study was conducted among 45 city bus drivers aged 31-58 years (43.7 \pm 7.9$)$, seniority as driver 3-34 years (14.7 \pm 8.6$)$. Data on climate conditions (ambient temperature, air pressure, humidity, wind speed, precipitations) on particular study days was obtained from the Institute of Meteorology and Water Management, National Research Institute Warsaw, Poland. Fatigue was assessed using the Fatigue Assessment Questionnaire, developed at Nofer Institute of Occupational Medicine (Lodz, Poland). The total level of fatigue was significantly $(p=0.045)$ higher after driving on HTR than on MR. The number of symptoms was also significantly higher $(p<0.05)$ among drivers working on HTR. After MR, significant correlations were found between wind speed and heavy eyelid feeling, being prone to forgetting, eye strain, frequent blinking, and between ambient temperature and feeling thirsty. After HTR feeling thirsty, tiredness and difficulty in making decisions correlated with ambient temperature and feeling thirsty with wind speed. Climate conditions can modify the drivers fatigue; therefore, we should be aware of their impact on well-being.
\end{abstract}

Keywords Climate conditions $\cdot$ Bus drivers $\cdot$ Symptoms of fatigue $\cdot$ Safe driving $\cdot$ Road accident

\section{Introduction}

In view of the climate changes related to global warming, the problems of heat exposure and its health consequences for the working population have been gaining interest (Kjellstrom et al. 2017; Błażejczyk et al. 2014). High ambient temperature and humidity were found to have a significant impact on workability, capacity and labour productivity of blue collar workers in outdoor jobs as well as on general work ability in other occupations (Brode et al. 2017). Increased ambient temperature affects also the safety of work. The rate of risky behaviours at work was reported to increase when ambient air temperature was

Alicja Bortkiewicz

alab@imp.lodz.pl

1 Department of Work Physiology and Ergonomics, Nofer Institute of Occupational Medicine, 8 Teresy St., 91-348 Lodz, Poland

2 Institute of Meteorology and Water Management, National Research Institute, 61 Podlesna St., 01-673 Warsaw, Poland either higher or lower than the thermal comfort conditions $\left(17^{\circ}-23^{\circ}\right.$ WBGT-Wet Bulb Globe Temperature) (Ramsey et al. 1983) determined for the general majority of workers. High ambient temperature is a recognised risk factor of traffic accidents, which was confirmed by the findings from a number of studies conducted across countries of moderate and tropical zones (Morabito et al. 2006; Nofal and Saeed 1997). When ambient temperature was included in the risk factor analysis of fatal traffic accidents, it was found to be more significant than such factors as alcohol intoxication or unfastened seat belts (Zlatoper 1991). It was also associated with the sensation of malaise and increasing drivers fatigue which may be an indirect cause of traffic accidents (Fujii et al. 2015; Noelke et al. 2016).

Drivers' fatigue was reported to be an important risk factor in most traffic crashes. Statistics show that drivers' fatigue accounts for about 10 to $15 \%$ of heavy road traffic accidents (RTA). The probability of participating in a traffic accident for a tired driver is eight times as high as for a well-rested driver. In this context, drivers' fatigue and the resulting accidents have been subject to extensive research (Thiese et al. 2015). 
Professional driving is known to be burdened with several risk factors for fatigue, including night time work, long hours of working, improper conditions of relaxation, prolonged time spent in sedentary posture, and a high level of responsibility for the passengers and the cargo. Among these factors, the climate also plays an important role, especially the temperature, as there is a proven relationship between the thermal load and the functioning of the organism (Enander 1989). According to various studies the work capacity reduces as the WBGT values exceeds $26-30{ }^{\circ} \mathrm{C}$ (Kjellstrom et al. 2009). The deterioration of physiological functions under environmental conditions exceeding the level of thermal comfort has a significant impact on the drivers' performance and productivity. When scientists compared driving a motor vehicle for a distance of $600 \mathrm{~km}$ at conditions of thermal comfort $\left(24{ }^{\circ} \mathrm{C}\right)$ and at heat stress $\left(37^{\circ} \mathrm{C}\right)$, the drivers' performance was found to decrease by $35 \%$ on average as the temperature increased. The drivers' performance was measured as the number of errors in driving technique and incidental changes of road lane during driving (Mackie and O'Hanlon 1977).

In the study by Wyon et al., the drivers exposed to heat stress showed increased heart rate and a higher level of fatigue as the time of driving increased (Wyon et al. 1996). In another experiment when the drivers' vigilance during driving at $27^{\circ} \mathrm{C}$ was examined (in terms of response to random light stimuli by pressing a button when the light appeared), the number of omitted responses increased by as much as $50 \%$ compared to control conditions $\left(21^{\circ} \mathrm{C}\right)$ Hancock and Vasmatzidis (2003). When the drivers' performance at $35^{\circ} \mathrm{C}$ and $20^{\circ} \mathrm{C}$ was evaluated, the lateral lane position variability was found to be by $13 \%$ higher for driving at $35{ }^{\circ} \mathrm{C}$ than at $20^{\circ} \mathrm{C}$ (Daanen et al. 2003).

The authors demonstrated that the rate of risky behaviours among drivers increased when ambient temperature was higher or lower than that preferred by most workers (Ramsey et al. 1983). The results of analyses of the risk factors for RTA revealed that the highest risk was noted on the days when mean ambient temperature varied from 24.8 to $27.5^{\circ} \mathrm{C}$ (Bergel-Hayat et al. 2013). High ambient temperature is a recognised risk factor increasing the RTA rate, which has been documented by the findings of studies conducted in the countries of moderate and tropical climate zones (Morabito et al. 2006; Nofal and Saeed 1997). When ambient temperature was included in the models analysing risk factors for fatal RTA, it was found to be more significant than the driver's alcohol consumption or unfastened seat belts (Zlatoper 1991).

It is not only the temperature, but the weather conditions in general that determine traffic safety (Theofilatos and Yannis 2014). Among the weather characteristics analysed most frequently are fog and rainfall or snowfall. The authors who investigated the risk of RTA as related to precipitation, point out that an impaired visibility due to rainfall is a more significant factor than a decreased wet road surface. This is confirmed by the findings that RTA rate rises only during the rain and not afterwards (Andrey and Yagar 1993). However, driving the vehicle moving on a wet road surface increases the level of the driver's fatigue (Chung et al. 2005).

Studies on the effects of weather conditions on drivers' performance have rarely considered the factor of atmospheric air pressure. In a Korean study, it was included as confounder in the analysis of a correlation between the rate of traumas from RTA and ambient temperature. The findings revealed a positive correlation between atmospheric air pressure and the rate of RTA-related traumas (Lee et al. 2014).

The studies reported above documented the influence of climate conditions on the drivers performance based on measuring physiological parameters and the drivers' reliability and driving quality. However, such studies are not only costly (as they require advanced instrumentation) but also exhaustive for the persons examined. A direct predictor for the driver that his work performance may worsen is the feeling of fatigue he is experiencing. The correlations between the subjective intensity of fatigue and the drivers' performance parameters are so high that the sensation of fatigue indicates an increasing risk of road accident (Smith 2016). The possible associations between climate parameters and drivers' fatigue have not been subject to specific studies thus far. Therefore, we decided to undertake a study to investigate whether and to what extent the particular climate parameters are related to fatigue perception by motor vehicle drivers. The tested hypothesis is that weather conditions (temperature, air humidity, rainfall, wind) affect the development of fatigue, even though the driver performs work in an air-conditioned cabin.

\section{Materials and methods}

The study was conducted among 45 city bus drivers in a large urban agglomeration in Poland. The subjects' age ranged 3158 years (mean $43.7 \pm 7.9$ lat), and the duration of working as driver varied from 3 to 34 years ( $14.7 \pm 8.6$ years). The participants were volunteers from a municipal bus company, with current medical certification qualifying for professional driving.

The drivers were informed about the study objectives and gave their written consent to participate in the project. The study protocol was approved by the Regional Bioethics Commission of the Nofer Institute of Occupational Medicine in Lodz.

\section{Study protocol}

The study was performed over the period lasting from July to October 2012. Each driver was examined four times before and after workshift on a monotonous route with a small traffic intensity outside the city center (MR), and before and after workshift on a heavy traffic route in the city center (HTR). 
The routes were classified by experts either as 'easy' or 'difficult' according to the following criteria: routing, traffic intensity and mean number of passengers. On the days of the study, the drivers started working at about 1 p.m., and their working time was slightly over $5 \mathrm{~h}$. This was the so-called short shift which is routine in the driver's daily schedule and enables a smooth flow of public transport during 'rush hours'. The study drivers had different routes to cover which was consistent with their individual work schedules. The selection of a short shift for the study was made with a view of standardising the time of the drivers' examinations.

\section{Local climate assessment}

Data on climate conditions (air temperature and pressure, humidity, wind speed, precipitations) on particular study days was collected from a local meteorological station in Lodz, affiliated to the Institute of Meteorology and Water Management, National Research Institute Warsaw, Poland. The data, recorded on hourly basis, for locations in the nearest vicinity of the bus routes driven by the study drivers, made it possible to calculate mean values for particular weather parameters for the working hours of each driver examined. This referred to air temperature and pressure, humidity and wind speed. We used meteorological data, because the cabin temperature was regulated by the drivers themselves as they set up the air conditioning system at the level that was most comfortable to them. The cabin was isolated from the passengers' area. Therefore, the individually regulated air temperature in the cabin was not a modifying factor of the drivers' fatigue. The driver has contact with outdoor temperature only during intervals between particular bus rides (about eight 10-min intervals during workday, i.e. $80 \mathrm{~min} /$ workshift).

The mean values regarding precipitation were calculated based on its daily total.

\section{Drivers' fatigue assessment}

To measure the level of drivers' fatigue, the Fatigue Assessment Questionnaire (FAQ) was used. The questionnaire was developed at the Work Physiology and Ergonomics Department of NIOM (Lodz, Poland) based on the inventory of 30 symptoms of fatigue prepared by the Research Committee on Industrial Fatigue, Japan Society for Occupational Health (Yoshitake 1978) and 10 symptoms of visual fatigue. The symptoms were classified into three groups: physical fatigue (17 symptoms: fatigue, feeling thirsty, yawning, tiredness, a need to take breaks, desire to lying down, legs fatigue, drowsiness, hands fatigue, malaise, feeling of heaviness, hand tremor, feeling of confusion in the mind, stiffness, awkwardness, dizziness, difficulty in breathing, uncertainty in maintaining standing position. Mental fatigue (11 symptoms: tendency to irrelevant thoughts, impatience, lack of willingness to work, lack of commitment to work, impaired attentiveness, apathy, being prone to making mistakes, being prone to forgetting, difficulty in thinking, difficulty in concentrating on work, difficulty in decisionmaking). Visual fatigue (12 symptoms-: frequent blinking, squinting, headache, eye strain, eye pain, lacrimation, heavy eyelids feeling, impaired visual acuity, eye burning sensation, stinging sensation, accommodation problems, blurred vision, glare impression. Each symptom was assessed using a fivepoint scale: (0) not at all, (1) somewhat, a little, slightly, (2) moderately, (3) fairly much/pretty much and (4) very much. For each driver, the total fatigue score was calculated. This could range from 0 to 160 , with a higher score indicating a more intense fatigue. In addition, the participants completed a short questionnaire to elicit information on the length of night sleep, the time of being active before work, and the time and type of meal consumed before the examination.

\section{Statistical analysis}

Statistical analysis included Student's $t$ test for independent variables for comparisons of the level of fatigue after driving on the monotonous and heavy traffic routes. Correlation coefficients were calculated to check the relationship between climate parameters and the symptoms of fatigue (significance level $\alpha=0.05$ ). For the statistical analysis, Statistica 8.0 was used.

\section{Results}

Analysis of the parameters that can modify the level of drivers' fatigue revealed no significant differences at the start of work shift for the drivers working on the monotonous (MR) or heavy traffic (HTR) bus route. The times of the end of workshift and the overall working time were also very similar for each route. Detailed data are shown in Table 1.

\section{Climate assessment}

Data on climate parameters during the drivers' work shifts are presented in Table 2. The climate conditions did not differ significantly for the MR and HTR. During the study period, no extreme weather conditions were recorded.

\section{Drivers' fatigue assessment}

The total level of fatigue was found to be significantly ( $p=$ $0.045)$ higher after driving on HTR $(8.49 \pm 9.30)$ than on MR (6.27 \pm 7.68$)$. After a working day spent on MR, the drivers reported $5.42 \pm 6.43$ fatigue symptoms (range $0-21$ ) on average. The number of symptoms was significantly higher, 6.87 \pm 6.69 (range $0-24$ ), among drivers working on HTR. The 
Table 1 Characteristics of parameters modifying the level of drivers' fatigue (mean, SD, range)

\begin{tabular}{llll}
\hline & Monotonous route & Heavy traffic route & $p$ \\
\hline Duration of sleep on the night preceding examination (h) & $7.95 \pm 1.60(4.0-12.0)$ & $8.20 \pm 1.48(4.75-12.0)$ & $12: 22-14: 14$ \\
Start of work shift (h) & $12: 10-14: 57$ & $17: 00-19: 35$ & .151 \\
End of work shift (h) & $16: 55-19: 43$ & $323 \pm 40(240-400)$ & .480 \\
Duration of work shift (min) & $314 \pm 41(183-386)$ & .319 \\
\hline
\end{tabular}

rating of the specific symptoms of fatigue after driving on HTR and MR is presented in Fig. 1.

Most of the symptoms were found to be more intense after a working day spent on HTR than on MR; for seven symptoms, these differences were statistically significant. After a workshift on HTR, the most common symptom was a general feeling of fatigue (22 drivers, $48.9 \%$ ), feeling of thirst (20 drivers, $44.4 \%$ ), tendency to irrelevant thoughts (20 drivers, 44.4\%), yawning (16 drivers, 35.6\%) and tiredness (16 drivers, 35.6\%). After driving on MR, the most frequently reported symptoms were the general feeling of fatigue (20 drivers, 44.4\%), tendency to irrelevant thoughts (18 drivers, 40.0\%), feeling thirsty (16 drivers, $35.6 \%$ ), and legs fatigue (15 drivers, 33.3\%).

Among drivers working on HTR, the total fatigue and seven individual symptoms of fatigue correlated with the duration of work shift. In workers driving on MR, the working time was found to have a lower impact on fatigue. After MR shift, only two individual symptoms of fatigue (difficulty in concentrating on work and sleepiness), but not the total fatigue, were found to correlate with the duration of work shift (Table 3).

The ratings for some fatigue symptoms after work shift were found to correlate with specific climate parameters. Much more significant correlations between climate conditions and fatigue could be noted after working on MR than HTR. Detailed data are presented in Table 4. For workers driving on HTR, the feeling thirsty and tiredness correlated with ambient air temperature. A negative correlation was found between these symptoms and the difficulty in decision-making. Moreover, the feeling thirsty negatively correlated with wind speed. None of the symptoms of fatigue correlated with atmospheric air pressure or humidity. After MR workshift, two symptoms were significantly more intense during precipitation: difficulty in thinking $(p=0.046)$ and difficulty in concentrating on work $(p=0.046)$ After HTR workshift, only tendency to irrelevant thoughts $(p=0.049)$ was more intense during precipitation.

\section{Discussion}

The findings of the present study revealed that the level of drivers' fatigue was generally low and amounted to about $10 \%$ and $13 \%$ of the maximum rating for the total fatigue after working on MR and HTR, respectively. The underlying reason could be the relatively short working hours; however, this was found to be less important among drivers working on MR. It can be assumed that longer working hours could be associated with increased fatigue, especially among drivers working on HTR. We presume that relatively low scores for particular symptoms may be attributed also to the good adaptation of the drivers to their job.

The climate conditions as recorded for the study period can be defined as neutral (temperature about $20^{\circ} \mathrm{C}$, moderate humidity, atmospheric air pressure about $993 \mathrm{hPa}$ and no fog) and no extreme weather conditions were noted. The influence of climate on overall drivers' fatigue was rather low but

Table 2 Characteristics of climate parameters on the drivers' working days

\begin{tabular}{|c|c|c|c|}
\hline Climate parameters & Monotonous route & Heavy traffic route & $p$ \\
\hline \multicolumn{4}{|c|}{ Ambient air temperature $\left({ }^{\circ} \mathrm{C}\right)$} \\
\hline Mean & 19.61 & 19.94 & \multirow[t]{4}{*}{.789} \\
\hline SD & 6.00 & 5.45 & \\
\hline Median & 19.24 & 20.57 & \\
\hline Range & $3.2-31.0$ & $8.6-28.8$ & \\
\hline \multicolumn{4}{|c|}{ Atmospheric air pressure $(\mathrm{hPa})$} \\
\hline Mean & 993.2 & 992.28 & \multirow[t]{4}{*}{.352} \\
\hline SD & 5.42 & 3.8 & \\
\hline Median & 994.47 & 992.03 & \\
\hline Range & $983.3-1004.9$ & $982.4-998.8$ & \\
\hline \multicolumn{4}{|l|}{ Humidity (\%) } \\
\hline Mean & 58.48 & 60.86 & \multirow[t]{4}{*}{.453} \\
\hline $\mathrm{SD}$ & 16.21 & 13.48 & \\
\hline Median & 57.57 & 61.14 & \\
\hline Range & $32.3-98.6$ & $35.3-93.3$ & \\
\hline \multicolumn{4}{|l|}{ Wind speed $(\mathrm{m} / \mathrm{s})$} \\
\hline Mean & 3.75 & 3.81 & \multirow[t]{4}{*}{.822} \\
\hline SD & 1.15 & 1.32 & \\
\hline Median & 3.86 & 3.57 & \\
\hline Range & $1.5-7.0$ & $1.6-6.7$ & \\
\hline \multicolumn{4}{|l|}{ Precipitation (mm) } \\
\hline Mean & 2.24 & 1.13 & \multirow[t]{4}{*}{.155} \\
\hline SD & 4.64 & 2.35 & \\
\hline Median & 0 & 0 & \\
\hline Range & $0-20.9$ & 0-98 & \\
\hline
\end{tabular}


Fig. 1 Ratings for specific fatigue symptoms after driving on monotonous (MR) and heavy traffic (HTR) route (mean and standard error). *Statistically significant differences between assessment of symptoms after MR and HTR

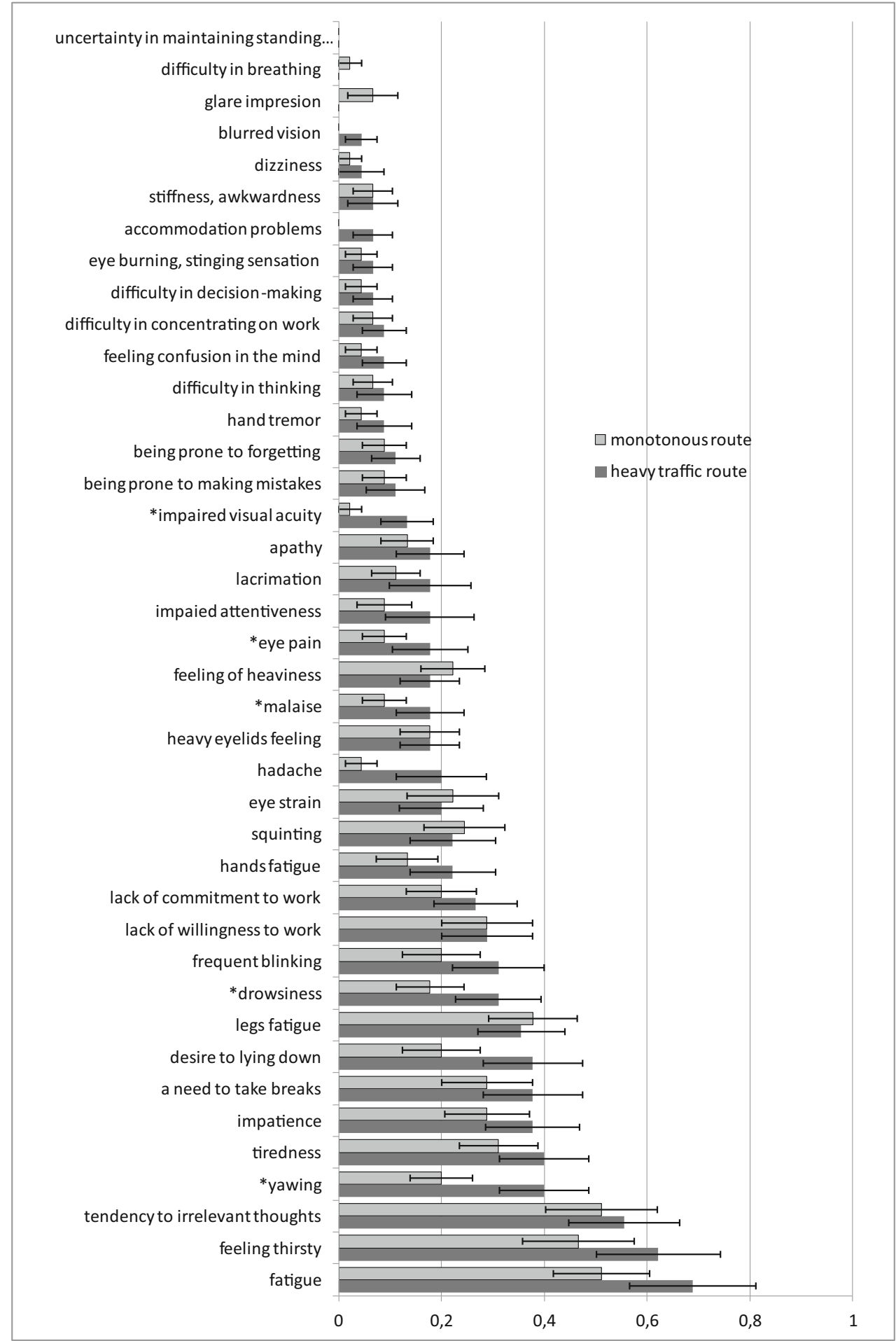

* statistically significant differences between assessment of symptom after MR and HTR showing a positive correlation with fatigue symptoms. As the weather conditions were becoming more intense and an increase was noted in ambient air temperature, pressure and humidity as well as wind speed, the sensation of the symptoms of fatigue was getting more intense. The fact that the increase in the intensity of fatigue symptoms in response to weather conditions was relatively small, can be attributed to the rather small range of climate variables, and to the high level of competence and job experience of the drivers examined. It has been shown that people with high qualifications and skills in 
Table 3 Relationship between duration of work shift for drivers working on a monotonous route (MR) or heavy traffic route (HTR) and drivers' fatigue ( $r$ Pearson's correlation coefficient)

\begin{tabular}{|c|c|c|c|c|}
\hline \multirow[t]{2}{*}{ Fatigue symptoms } & \multicolumn{2}{|c|}{ Monotonous route } & \multicolumn{2}{|c|}{ Heavy traffic route } \\
\hline & $r$ & $p$ & $r$ & $p$ \\
\hline Total fatigue & & & .357 & .016 \\
\hline Difficulty in concentrating on work & .302 & .044 & & \\
\hline Drowsiness & -.488 & .001 & .326 & .029 \\
\hline A need to take breaks & & & .364 & .014 \\
\hline Desire to lie down & & & .347 & .020 \\
\hline Eye strain & & & .381 & .010 \\
\hline Eye pain & & & .367 & .013 \\
\hline Headache & & & .353 & .018 \\
\hline Tiredness & & & .353 & .018 \\
\hline
\end{tabular}

certain activities are capable of maintaining high levels of performance despite the effects of heat stress (Hancock 1986).

In order to assess the current and future potential workplace heat exposures and to develop policies for prevention of heat related health threats and losses of workability, which is an alternative term to work capacity and an input into labour productivity, it is important to have scientifically based heat stress indicators available.

Our study revealed a significant correlation between some symptoms of fatigue and ambient air temperature, wind speed and total rainfall during work shift. In our study, wind speed outside had influence on five symptoms of fatigue after MR. The blowing wind has a direct impact on driving and is perceived by the driver holding a steering wheel as he has to control the vehicle's stability on the road. The wind gust of $5 \mathrm{~m} / \mathrm{s}$ was found to be equal to $300 \mathrm{~N}$ (Reński 2001). According to Bos et al., wind blowing may have also influence on the driver's feeling of comfort and physical wellbeing (Bos et al. 2012).

The incidents of rainfall influenced on difficulty in thinking and difficulty in concentrating on work during driving on monotonous route and tendency to irrelevant thoughts during work on heavy route. In our findings, this was most evident during MR driving. Other researchers investigating the risk of road traffic accidents as related to
Table 4 Relationship between symptoms of fatigue after work and climate conditions during work (statistically significant Pearson's correlation coefficients)

\begin{tabular}{|c|c|c|c|c|}
\hline \multirow[t]{2}{*}{ Symptoms of fatigue } & \multicolumn{2}{|c|}{ Ambient temperature } & \multicolumn{2}{|c|}{ Wind speed } \\
\hline & $r$ & $p$ & $r$ & $p$ \\
\hline \multicolumn{5}{|l|}{ Monotonous route } \\
\hline Feeling of thirsty & 0.301 & 0.045 & & \\
\hline Being prone to forgetting & & & 0.452 & $0.002 *$ \\
\hline \multicolumn{5}{|l|}{ Feeling of confusion in the mind } \\
\hline Heavy eyelid feeling & & & 0.401 & $0.006^{*}$ \\
\hline Eye strain & & & 0.341 & $0.022 *$ \\
\hline Frequent blinking & & & 0.319 & $0.033 *$ \\
\hline \multicolumn{5}{|l|}{ Impaired visual acuity } \\
\hline \multicolumn{5}{|l|}{ Difficulty in decision-making } \\
\hline \multicolumn{5}{|l|}{ Difficulty in thinking } \\
\hline \multicolumn{5}{|c|}{ Difficulty in concentrating on work } \\
\hline Number of fatigue symptoms & & & 0.310 & $0.038 *$ \\
\hline \multicolumn{5}{|l|}{ Heavy traffic route } \\
\hline Feeling thirsty & 0.343 & 0.021 & -0.297 & $0.048 *$ \\
\hline Tiredness & 0.321 & 0.032 & & \\
\hline Difficulty in making decisions & -0.321 & 0.032 & & \\
\hline
\end{tabular}

* Statistical significance 
precipitation point out that an impaired visibility due to rainfall is a more significant risk factor than a decreased wet road surface. This is supported by the findings indicating that the accident rate increases only during rainfall and not afterwards (Theofilatos and Yannis 2014, Andrey and Yagar 1993).

In general, the worsening of weather conditions during driving on MR was associated with an intensification of passive fatigue symptoms among drivers (Desmond and Hancock 2001).

With regard to drivers working on HTR, the rising ambient air temperature contributed to more intense sensation of tiredness but did not have much influence on decision-making. Johnson and Kobbrick pointed out that high temperature was associated with deteriorated work performance during monotonous, repetitive and boring tasks, but low impact was noted for the tasks that were interesting or challenging (Johnson and Kobrick 2001). A similar relationship seems to apply to fatigue which is an indicator of a subjective feeling of impaired performance.

Irrespective of the type of bus route the drivers were working, the higher levels of ambient temperature correlated with the feeling of thirst among drivers.

Thirst is an unpleasant feeling and a symptom of insufficient hydration of the organism (Armstrong et al. 2014). However, it can be felt only when the systemic dehydration reaches the level of $1-2 \%$ of body mass. At this level, the drivers experience not only malaise and increased fatigue but also concentration problems and deficient performance of tasks that require attention, psychomotor skills and direct memory (Shirreffs et al. 2004; EuroHEAT. Technical report, 2009; Adan 2012). Moreover, body dehydration resulting from heat stress leads to decreased vigour and intensifies the feeling of fatigue (Wetsel 2011). Therefore, the feeling of thirst can be regarded as an indirect indicator of impaired performance which can be particularly significant for the drivers' safety. Appropriate supplementation of liquids is also important for the drivers since water intake increases the potential to reduce sleepiness (Pross et al. 2014). In Poland, there are no specific regulations with regard to drinking limitations for bus drivers, but the drivers themselves tend to limit the drinking as they want to avoid the use of public toilets.

To the best of our knowledge, there have been no studies investigating the impact of climate conditions (except for cabin climate parameters) on the drivers' fatigue and related consequences. The authors assumed that ambient climate conditions may modify the perception of work-related fatigue by the drivers. An example of the influence the external conditions on work performance and the feeling of fatigue are the findings by Bos et al. (2012) which 'reveal subtle environmental influence on psychopathology'. Although these findings concerned a patient diagnosed with recurrent anxiety disorder, one cannot exclude a possibility that they may also apply to healthy individuals.

Moreover, there are also some literature reports on the influence of meteorological environment on human health and wellbeing (Ezekowitz et al. 2013, Wang et al. 2015, Yackerson et al. 2012). Seeing that we have practically no control over the weather conditions to which professional drivers are exposed during work, except for the cabin temperature which the driver can regulate, we should realise what effect they can have on the drivers' performance and wellbeing. The relationship between climate conditions and drivers' fatigue observed in our study may get stronger as the duration of work increases and the fatigue becomes more intense. These preliminary findings point to a necessity for further in-depth studies to elucidate the above.

Acknowledgments We want to thank Ewa Brejnakowska for her assistance in developing the revised version of the manuscript.

Funding information This work was supported by Innovative Economy Programme: Priority 1 . Research and development of modern technologies activity (UDA-POIG.01.03.01-10-085/09) AND PROJECT PBS3 / B9 / 29/2015, CHIEF INVESTIGATOR: PROF. DR HAB N. MED. ALICJA BORTKIEWICZ.

\section{Compliance with ethical standards}

The study protocol was approved by the Regional Bioethics Commission of the Nofer Institute of Occupational Medicine in Lodz.

Open Access This article is distributed under the terms of the Creative Commons Attribution 4.0 International License (http:// creativecommons.org/licenses/by/4.0/), which permits unrestricted use, distribution, and reproduction in any medium, provided you give appropriate credit to the original author(s) and the source, provide a link to the Creative Commons license, and indicate if changes were made.

\section{References}

Adan A (2012) Cognitive performance and dehydration. J Am Coll Nutr 31:71-78

Andrey J, Yagar S (1993) A temporal analysis of rain-related crash risk. Accid Anal Prev 25:465-472

Armstrong LE, Ganio MS, Klau JF, Johnson EC, Casa DJ, Maresh CM (2014) Novel hydration assessment techniques employing thirst and a water intake challenge in healthy men. Appl Physiol Nutr Metab 39:138-144. https://doi.org/10.1139/apnm-2012-0369

Bergel-Hayat R, Debbarh M, Antoniou C, Yannis G (2013) Explaining the road accident risk: weather effects. Accid Anal Prev 60:456465. https://doi.org/10.1016/j.aap.2013.03.006

Błażejczyk K, Baranowski J, Błażejczyk A (2014) Heat stress and occupational health and safety - spatial and temporal differentiation. Miscellanea Geographica - Regional Studies on Development, 18. https://doi.org/10.2478/mgrsd-2014-0011 
Bos EH, Hoenders R, de Jonge P (2012) Wind direction and mental health: a time-series analysis of weather influences in a patient with anxiety disorder. BMJ Case Rep 2012. https://doi.org/10.1136/bcr2012-006300

Brode P, Fiala D, Lemke B, Kjellstrom T (2017) Estimated work ability in warm outdoor environments depends on the chosen heat stress assessment metric. Int J Biometeorol. https://doi.org/10.1007/s00484$017-1346-9$

Chung E, Ohtani O, Warita H, Kuwahara M, Morita H (2005) Effect of rain on travel demand and traffic accidents. In: Proc. IEEE Conf. on Intelligent Transportation Systems pp. 1080-1083

Daanen HA, van de Vliert E, Huang X (2003) Driving performance in cold, warm, and thermoneutral environments. Appl Ergon 34:597602

Desmond PA, Hancock PA (2001) Active and passive fatigue states. In: Hancock PA, Desmond PA (eds) Stress, workload, and fatigue. Lawrence Erlbaum Associates, New Jersey, pp 455-465

Enander AE (1989) Effects of thermal stress on human performance. Scand J Work Environ Health 15(supl.1):27-33

EuroHEAT (2009) Technical report. Improving public health responses to extreme weather/heat-waves. WHO Regional Office for Europe, Copenhagen

Ezekowitz JA, Bakal JA, Westerhout CM, Giugliano RP, White H, Keltai M, Prabhakaran D, Tricoci P, Van de Werf F, Califf RM, Newby LK, Armstrong PW (2013) The relationship between meteorological conditions and index acute coronary events in a global clinical trial. Int J Cardiol 168:2315-2321. https://doi.org/10.1016/j.ijcard.2013. 01.061

Fujii H, Fukuda S, Narumi D, Ihara T, Watanabe Y (2015) Fatigue and sleep under large summer temperature differences. Environ Res 138: 17-21. https://doi.org/10.1016/j.envres.2015.02.006

Hancock PA (1986) The effect of skill on performance under an environmental stressor. Aviat Space Environ Med 57:59-64

Hancock PA, Vasmatzidis I (2003) Effects of heat stress on cognitive performance: the current state of knowledge. Int J Hyperth 19: 355-372

Johnson RF, Kobrick JL (2001) Psychological aspects of military performance in hot environment. In: Kent B, Pandolf R, Burr E (eds) Medical aspects of harsh environments. National Academy Press, Washington DC, pp 135-159

Kjellstrom T, Holmer I, Lemke B (2009) Workplace heat stress, health and productivity - an increasing challenge for low and middleincome countries during climate change. Glob Health Action 2(1): 2047. https://doi.org/10.3402/gha.v2i0.2047

Kjellstrom T, Freyberg C, Lemke B, Otto M, Briggs D (2017) Estimating population heat exposure and impacts on working people in conjunction with climate change. Int J Biometeorol 62:291-306. https:// doi.org/10.1007/s00484-017-1407-0

Lee WK, Lee HA, Hwang SS, Kim H, Lim YH, Hong YC, Ha EH Park H (2014) A time series study on the effects of cold temperature on road traffic injuries in Seoul, Korea. Environ Res 132:290-296. https:// doi.org/10.1016/j.envres.2014.04.019
Mackie RR, O'Hanlon JF (1977) A study of the combined effect of extended driving and heat stress on driver arousal and performance. In: Mackie RR (ed) Vigilance: theory, operational performance and physiological correlates. Plenum Press, New York, pp 537-558

Morabito M, Cecchi L, Crisci A, Modesti PA, Orlandini S (2006) Relationship between work-related accidents and hot weather conditions in Tuscany (Central Italy). Ind Health 44:458-464

Noelke C, McGovern M, Corsi DJ, Jimenez MP, Stern A, Wing IS, Berkman L (2016) Increasing ambient temperature reduces emotional well-being. Environ Res 151:124-129. https://doi.org/10. 1016/j.envres.2016.06.045

Nofal FH, Saeed AA (1997) Seasonal variation and weather effects on road traffic accidents in Riyadh city. Public Health 111:51-55

Pross N, Demazières A, Girard N, Barnouin R, Metzger D, Klein A, Perrier E, Guelinckx I (2014) Effects of changes in water intake on mood of high and low drinkers. PLoS One 9:e94754. https:// doi.org/10.1371/journal.pone.0094754

Ramsey JD, Burford CL, Beshir MY, Jensen RC (1983) Effects of workplace thermal conditions on safe behavior. J Saf Res 14:105-114

Reński A (2001) Identification of driver model parameters. Int J Occup Saf Ergon 7:79-90

Shirreffs SM, Merson SJ, Fraser SM, Archer DT (2004) The effects of fluid restriction on hydration status and subjective feelings in man. Br J Nutr 91:951-958

Smith AP (2016) A UK survey of driving behaviour, fatigue, risk taking and road traffic accidents. BMJ Open 6(8):e011461. https://doi.org/ 10.1136/bmjopen-2016-011461

Theofilatos A, Yannis G (2014) A review of the effect of traffic and weather characteristics on road safety. Accid Anal Prev 72:244 256. https://doi.org/10.1016/j.aap.2014.06.017

Thiese MS, Ott U, Robbins R, Effiong A, Murtaugh M, Lemke MR, Deckow-Schaefer G, Kapellusch J, Wood E, Passey D, Hartenbaum N, Garg A, Hegmann KT (2015) Factors associated with truck crashes in a large cross section of commercial motor vehicle drivers. J Occup Environ Med 57:1098-1106. https://doi. org/10.1097/JOM.0000000000000503

Wang C, Fang L, Cao W, Zhang Y, Cao K, Xu Q, Guo X (2015) Analysis on impact of meteorological factors on incidence of hand, foot and mouth disease based on Bayes spatial-temporal theory. Zhonghua Liu Xing Bing Xue Za Zhi 36:476-480

Wetsel WC (2011) Hyperthermic effects on behavior. Int J Hyperth 27: 353-373. https://doi.org/10.3109/02656736.2010.550905

Wyon DP, Wyon I, Norin F (1996) Effects of moderate heat stress on driver vigilance in a moving vehicle. Ergonomics 39:61-75

Yackerson NS, Bromberg L, Adler B, Aizenberg A (2012) Possible effects of changes in the meteorological state over semi-arid areas on the general well-being of weather-sensitive patients. Environ Health 11:26. https://doi.org/10.1186/1476-069X-11-26

Yoshitake H (1978) Three characteristic patterns of subjective fatigue symptoms. Ergonomics 21:231-233. https://doi.org/10.1080/ 00140137808931718

Zlatoper TJ (1991) Determinants of motor vehicle deaths in the United States: a cross-sectional analysis. Accid Anal Prev 23:431-436 